\title{
Projektübergreifende Unterstützung von Registern in Entwicklung, Umsetzung und Betrieb
}

\section{Cross-Project Support of Registries in Development, Implementation and Operation}

\author{
Autoren \\ Sonja Harkener, Jürgen Stausberg
}

Institut

Institut für Medizinische Informatik, Biometrie und Epidemiologie, Universitätsklinikum Essen, Essen, Deutschland

\section{Schlüsselwörter}

Datenqualität, Metadaten, Register, Registerentwicklung, Registerstandards, Versorgungsforschung

\section{Keywords}

data accuracy, metadata, health registries, registry development, registry standards, health services research

\section{Bibliografie}

Gesundheitswesen 2021; 83 (Suppl. 1): S54-S59

DOI $10.1055 / a-1537-9324$

ISSN 0949-7013

(C) 2021. Thieme. All rights reserved. Georg Thieme Verlag KG, Rüdigerstraße 14, 70469 Stuttgart, Germany

\section{Korrespondenzadresse}

Sonja Harkener

Institut für Medizinische Informatik,

Biometrie und Epidemiologie

Universitätsklinikum Essen

Hufelandstraße 55

45122 Essen

Deutschland

sonja.harkener@uk-essen.de

\section{ZUSAMMENFASSUNC}

Ziel Vom Bundesministerium für Bildung und Forschung wurde ein Begleitprojekt zu einer Förderinitiative zum Aufbau modellhafter Register gefördert. Ziel war die registerübergreifende Unterstützung von zunächst 16, später 6 Projekten bezüglich methodischer, technischer und struktureller Standards.

Methodik Die 16 Projekte wurden zunächst bei der Konzeptentwicklung begleitet, u. a. durch eine Vorlage für ein Registerprotokoll. Außerdem wurde eine Expertenberatung organisiert und umgesetzt. Zur Unterstützung bei der Auswahl einer
IT-Lösung wurde ein Workshop in Form einer Challenge ausgerichtet, auf dem verschiedene Anbieter ihre Software für Register vorstellten. Die Merkmalskataloge der Projekte wurden in einen Metadaten-Katalog überführt und in das Standardmodell der ISO/IEC 11179 übertragen. Für ein in der Betriebsphase umzusetzendes, registerübergreifendes Qualitätsmanagement wurde ein Set von Qualitätsindikatoren festgelegt. Zur Verbesserung der Datenqualität waren die Indikatoren regelmäßig zu übermitteln und auszuwerten.

Ergebnisse Die Vorlage für ein Registerprotokoll wurde mehrheitlich von den Projekten für einen Antrag auf Förderung ihrer Betriebsphase genutzt. Auf dem Workshop zu IT-Lösungen wurden 12 Produkte für Registersoftware vorgestellt; die Projekte entschieden sich allerdings aus unterschiedlichen Gründen für andere Lösungen. Durch die Überführung der Merkmalskataloge in ein Standardmodell wurde ein Vergleich von Merkmalen und Wertemengen ermöglicht. Daraus konnten Empfehlungen für wichtige Elemente formuliert werden. Für das Qualitätsmanagement wurde ein Set von fünf Qualitätsindikatoren festgelegt, zu denen für das Jahr 2020 eine erste Auswertung erfolgte.

Schlussfolgerung Die Vorlage eines Registerprotokolls dient einer strukturierten Erarbeitung eines Konzepts. Die Verwendung eines einheitlich strukturierten Merkmalskatalogs unterstützt die Einhaltung der FAIR-Prinzipien. Eine Überwachung der Datenqualität kann durch eine regelmäßige, registerübergreifende Ermittlung von Qualitätsindikatoren erreicht werden.

\section{ABSTRACT}

Objective The German Federal Ministry of Education and Research funded a project accompanying a funding initiative for registries in health services research. The aim was to provide cross-registry support initially for 16 and later 6 projects with regard to methodological, technical and structural standards. Methods The 16 projects were initially guided in concept development, e. g., providing a template for a registry protocol. Furthermore, an expert consultation was organized and carried out. To assist in the selection of an IT solution, a challenge workshop was hosted where different vendors presented their software for registries. The catalogs of data elements of the 
projects were migrated into a metadata catalog and transferred to the standard model of ISO/IEC 11179. A set of quality indicators was defined for a cross-registry quality management approach to be implemented during the operational phase. To improve data quality, the indicators were to be transmitted and evaluated on a regular basis.

Results The template for a registry protocol was used by the majority of projects when applying for funding of their operational phase. At the workshop on IT solutions, 12 products for registry software were presented; however, the projects opted for other solutions for different reasons. Transferring the cata- logs of data elements into a standard model enabled a comparison of attributes and value sets, which in turn enabled formulation of recommendations for important elements. A set of five quality indicators was defined for quality management, for which an initial evaluation was carried out for 2020 .

Conclusion The template of a registry protocol serves a systematic development of a concept. The use of a uniformly structured catalog of data elements supports compliance with the FAIR principles. Monitoring of data quality can be achieved by regularly identifying quality indicators across registries.

\section{Einleitung}

Im Rahmen des Aktionsplans Versorgungsforschung wurde Ende des Jahres 2016 vom Bundesministerium für Bildung und Forschung (BMBF) eine Förderinitiative zum Aufbau modellhafter Register für die Versorgungsforschung ausgeschrieben. Es sollten neue, patientenbezogene Register zu wichtigen Fragen der Versorgungsforschung aufgebaut werden, die hohe Qualitätsstandards erfüllen und Modellcharakter haben. Hierfür erhielten 16 Projekte Ende 2017 eine 9-monatige Anschubfinanzierung für eine erste Phase (Konzeptentwicklungsphase), an deren Ende ein detailliertes Konzept zur Realisierung des Registers eingereicht werden sollte, insbesondere mit dem Nachweis einer Nachhaltigkeit über den Zeitraum der Förderung hinaus. In der sich anschließenden Realisierungsphase sollten die Register implementiert und in Betrieb genommen und die wissenschaftlichen Fragestellungen bearbeitet werden. Für diese zweite Phase, die im Frühjahr 2019 begann, qualifizierten sich sechs Projekte, fünf davon wurden für fünf Jahre, eines für drei Jahre finanziert.

Zeitgleich mit dem Beginn der Konzeptentwicklungsphase wurde ein Begleitprojekt zunächst für zwei Jahre gefördert. Dieses sollte die Projekte registerübergreifend bezüglich methodischer, technischer und struktureller Standards unterstützen. Das Begleitprojekt wurde in einer Kooperation zwischen der Technologie- und Methodenplattform für die vernetzte medizinische Forschung (TMF) und dem Institut für Medizinische Informatik, Biometrie und Epidemiologie (IMIBE) des Universitätsklinikums Essen mit Unterstützung durch das Deutsche Netzwerk Versorgungsforschung (DNVF) umgesetzt. Nach dem initialen Anschub wurde die Förderung des Begleitprojekts um weitere drei Jahre verlängert. Im Folgenden werden Methoden und Ergebnisse der projektübergreifenden Unterstützung der Register in Entwicklung, Umsetzung und Betrieb aus Sicht des Essener Teilprojekts dargestellt.

\section{Methodik}

\section{Konzeptentwicklungsphase}

In der 9-monatigen Konzeptentwicklungsphase lag ein Fokus der Arbeit des Begleitprojekts in der Unterstützung der Projekte bei der Erarbeitung der Konzepte, die am Ende der Phase für die Antragstellung zur Realisierungsphase eingereicht werden sollten (Konzeptberatung). Um ein Register erfolgreich zu betreiben ist eine gute Planung essentiell [1]. In der Förderinitiative wurde dazu zunächst eine Vorlage für ein Registerprotokoll entwickelt, welches analog zu einem Studienprotokoll bei klinischen Studien als Leitfaden für das jeweilige entstehende Register dienen sollte. Aus dem Memorandum Register für die Versorgungsforschung des DNVF [2] wurde eine Gliederung für ein solches Registerprotokoll übernommen, auf die Belange der Förderinitiative angepasst und mit den 16 Projekten abgestimmt. Am Ende der Konzeptentwicklungsphase wurde den Projekten die Möglichkeit vermittelt, einen externen Experten zur Begutachtung des Konzepts hinzuzuziehen. Dieser Experte wurde über ein Anmeldeverfahren per Internet bzw. durch aktives Ansprechen der Mitglieder der AG Register des DNVF ausgewählt und den Projekten über ein Zufallsverfahren zugeordnet.

Ein wichtiger Aspekt bei der Planung eines Registers liegt in der Festlegung der Softwarekomponenten. Die Projekte sollten insbesondere bei der Auswahl einer IT-Lösung für das Datenmanagement unterstützt werden. Zusätzlich zu den in der Literatur zugänglichen Übersichten zur Marktlage in Deutschland [3-5] sollten Softwarelösungen in Form einer Challenge vorgestellt werden. Dazu wurde ein 2-tägiger Workshop organisiert, auf dem Anbieter von Software für Register ihre IT-Lösungen den interessierten Projekten vorstellen konnten [6]. Dieses Vorgehen sollte die Projekte in die Lage versetzen, einen Einblick in den aktuellen Markt zu erhalten und damit Möglichkeiten und Grenzen der zurzeit verfügbaren Softwareunterstützung einzuschätzen. Um eine möglichst hohe Vergleichbarkeit der verschiedenen Softwarelösungen zu ermöglichen wurde im Vorfeld für den Ablauf eine Ausformulierung mit Anwendungsszenarien entworfen, außerdem wurden Gewichtungs-, Protokollierungs- und Bewertungsbögen erstellt. Die Anwendungsszenarien wurden einer Übersicht zu Anforderungen und Aufgaben von Kohorten und Registern entnommen (Projekt KoRegIT [7]). Die Ergebnisse wurden in einem Bericht zusammengestellt, an die Projekte versendet und im Verbund aus Begleitprojekt und Projekten diskutiert.

Ein wesentlicher Bestandteil bei der Planung eines Registers sind die Definitionen von Variablen und Wertemengen [1, 2, 8]. Um eine sekundäre Nutzungsmöglichkeit von Daten zu ermöglichen, sollten dabei die FAIR-Prinzipen - Findability, Accessibility, Interoperability, Reusability - beachtet werden [9]. Für den Aufbau eines MetadatenKatalogs der Förderinitiative sollten zunächst alle Merkmale der 16 Register erfasst werden. Um die Informationen in einem möglichst standardisierten Format zu erhalten, schlug das Begleitprojekt die 
Verwendung des Attributkatalogs von Leiner und Haux [10] als Format für die Datendefinitionen (z. B. Bezeichnungen, Wertemengen, Kodierungen) vor. Dazu wurde eine entsprechende Vorlage erstellt und mit allen Projekten abgestimmt (wahlweise in den Formaten Microsoft Access und Microsoft Excel). Ein Zweck des geplanten Metadaten-Katalogs sollte darin bestehen, die Verwendung von ähnlichen Merkmalen bei verschiedenen Projekten aufzudecken, Wertemengen und Kodierungen anzugleichen sowie die Verwendung von Standards zu fördern. Für die Erreichung dieser Ziele musste daher zunächst eine Möglichkeit zum Vergleich der unterschiedlichen Merkmalskataloge geschaffen werden. Dazu wurde eine Abbildung der Kataloge auf eine abstrakte Ebene vorgenommen, die es ermöglicht, Ähnlichkeiten und Abweichungen unabhängig von Schreibweisen und Modellierungsentscheidungen zu erkennen. Hierfür wurde auf einen internationalen Standard zurückgegriffen, die ISO/IEC 11179-3:2013(E) (Part 3: Registry metamodel and basic attributes) [11]. Die eingereichten Merkmalskataloge wurden in eine Access-Datenbank überführt und auf das Standardmodell der ISO/IEC 11179 übertragen [12].

Der Wert eines Registers hängt sehr von der Qualität der Daten $a b$, die durch entsprechende Verfahren optimiert werden sollte [13]. Für eine Unterstützung im Bereich des Qualitätsmanagements, insbesondere zur Überwachung und Verbesserung der Datenqualität, sollte ein Set von Qualitätsindikatoren in jedem Register der Förderinitiative implementiert werden. Die Kennzahlen dieser Qualitätsindikatoren sollten in der Betriebsphase für ein registerübergreifendes Benchmarking ausgewertet werden. Dieses Set war aus den 51 Qualitätsindikatoren der Leitlinie zum adaptiven Management von Datenqualität in Kohortenstudien und Registern [14] zusammenzustellen. Für die Auswahl wurde eine Umfrage unter den 16 Projekten im Web durchgeführt, in der jeder der 51 Qualitätsindikatoren zu sieben Kriterien bewertet wurde [15]. Das sich aus der Umfrage ergebene Set von Qualitätsindikatoren wurde im Nachgang technisch ausgearbeitet.

\section{Realisierungsphase}

Sechs Register konnten sich erfolgreich für die zweite Phase der Förderinitiative qualifizieren. Die Arbeit des Begleitprojekts fokussierte sich in dieser Phase u. a. auf die Bereiche Qualitätsmanagement, Metadaten und Modellhaftigkeit. $>$ Tab. 1 zeigt die Schwerpunkte der Projekte aus beiden Förderphasen.

Mit der Überführung der Merkmalskataloge in das Standardmodell der ISO/IEC 11179 hatte das Begleitprojekt die Möglichkeit, jedem der sechs Projekte eine Übersicht zu ähnlichen Elementen aus anderen Projekten der Konzeptentwicklungsphase mit abweichender Umsetzung im Merkmalskatalog zur Verfügung zu stellen. Ergänzend wurden aufgrund einer entsprechenden Umfrage des Begleitprojekts zu einigen Merkmalen Empfehlungen zu Wertemengen verfasst. Zwei Jahre nach der Zusammenführung der Merkmalskataloge der Konzeptentwicklungsphase wurden die für die Betriebsphase angepassten Merkmalskataloge der sechs verbliebenen Projekte ebenfalls dem Metadaten-Katalog der Förderinitiative hinzugefügt und entsprechend der ISO/IEC 11179 ergänzt.

Das mit allen Projekten aus der Konzeptentwicklungsphase festgelegte Set der Qualitätsindikatoren wurde mit den sechs verbliebenen Projekten nochmals diskutiert und auf ein sogenanntes
-Tab. 1 Medizinische Schwerpunkte der geförderten Projekte.

\begin{tabular}{|l|c|c|}
\hline Gebiet/Thema & $\begin{array}{c}\text { Konzept- } \\
\text { entwicklung }\end{array}$ & $\begin{array}{c}\text { Realisie- } \\
\text { rung }\end{array}$ \\
\hline Akute Erkrankungen & & \\
\hline Acute Respiratory Distress Syndrome & $\mathrm{x}$ & \\
\hline kindliches Fieber & $\mathrm{x}$ & $\mathrm{x}$ \\
\hline Herzinfarkt & $\mathrm{x}$ & \\
\hline Lungenembolie & $\mathrm{x}$ & \\
\hline $\begin{array}{l}\text { rezidivierende Steinerkrankungen des } \\
\text { oberen Harntraktes }\end{array}$ & $\mathrm{x}$ & $\mathrm{x}$ \\
\hline Chronische Erkrankungen & $\mathrm{x}$ & \\
\hline Zöliakie & $\mathrm{x}$ & $\mathrm{x}$ \\
\hline Querschnittslähmung & $\mathrm{x}$ & $\mathrm{x}$ \\
\hline nicht-infektiöse Uveitis & & \\
\hline Onkologie & $\mathrm{x}$ & \\
\hline metastasierendes Mammakarzinom & $\mathrm{x}$ & $\mathrm{x}$ \\
\hline $\begin{array}{l}\text { Prävention des erblichen Brust- und } \\
\text { Eierstockkrebses }\end{array}$ & $\mathrm{x}$ & \\
\hline Seltene Erkrankungen & $\mathrm{x}$ & \\
\hline Prader-Willi-Syndrom & $\mathrm{x}$ & \\
\hline systemischer Lupus erythematodes & & \\
\hline Maßnahmen & $\mathrm{x}$ & \\
\hline Knietotalendoprothese bei Osteoarthritis & & \\
\hline Impf-Informations-System & & \\
\hline Andere & & \\
\hline Nationales Mortalitätsregister & & \\
\hline lebende Nierenspender & & \\
\hline & & \\
\hline
\end{tabular}

Core-Set reduziert. In der Betriebsphase sollten die Kennzahlen jedes Qualitätsindikators regelmäßig an das Begleitprojekt übermittelt werden. Zur Sicherstellung von Transparenz und Vergleichbarkeit der Ergebnisse wurden über eine Vorlage projektspezifische Festlegungen erhoben. Diese Festlegungen wurden in Verfahrensanweisungen zusammengeführt sowie zu prüfende Zeiträume und Meldezeitpunkte festgelegt. Aus den Verfahrensanweisungen wurden Formulare zur Meldung der Ergebnisse aus den einzelnen Registern abgeleitet.

\section{Ergebnisse}

\section{Konzeptberatung}

Unter Verwendung bereits publizierter Empfehlungen wurde in Zusammenarbeit mit den Projekten eine Vorlage für ein Registerprotokoll erarbeitet, die von einem großen Teil der Projekte für die Einreichung ihres Antrags verwendet wurde. Die Vorlage war in Deutsch und Englisch verfügbar und steht öffentlich zur Verfügung (s. https://www.toolpool-gesundheitsforschung.de/). Die Ergebnisse wurden in das Update für das Memorandum Register für die Versorgungsforschung des DNVF eingebracht [16]. Die Beratung der Projekte und die Sichtung der Registerprotokolle durch externe Experten über eine schriftliche Stellungnahme, eine telefonische Erörterung oder ein Gespräch vor Ort wurde von 15 der 16 Projekte wahrgenommen. 


\section{IT-Lösungen für Register}

Auf dem Workshop zu IT-Lösungen stellten 12 Anbieter ihre Software für das Datenmanagement eines Registers vor. Alle Anbieter orientierten sich an dem vorgegebenen Ablaufplan, sodass für die teilnehmenden Vertreter aus 12 Projekten eine breite Übersicht über den Markt an Software zum Datenmanagement bei Registern erzielt werden konnte. Letztendlich hat sich allerdings keines der Projekte für eines der vorgestellten Softwareprodukte entschieden. Ein Projekt arbeitet komplett mit einer eigens für das Register entwickelten App, zwei Projekte entschieden sich für eine Open Source Lösung, ein Projekt entwickelte eine bestehende Softwarelösung weiter und ein Projekt startete eine Neuentwicklung. Das letzte Projekt bezieht seine Daten primär über die Infrastruktur der Medizininformatik-Initiative. Aktuell liegt die Unterstützung durch das Begleitprojekt in einem Austausch über Stärken und Schwächen der IT-Lösungen, so dass eine sachgerechte Weiterentwicklung der Produkte sowie eine den Möglichkeiten des Marktes angepasste Kommunikation mit den Anbietern erreicht wird.

\section{Metadaten}

Die vom Begleitprojekt zur Verfügung gestellte Vorlage für die Erfassung eines Merkmalskatalogs wurde von 13 Projekten verwendet; zwei Projekte übersandten ein Microsoft Word-Dokument. Von einem Projekt wurden keine Metadaten zur Verfügung gestellt. Die Vorlage für einen Merkmalskatalog steht öffentlich zur Verfügung (s. https://www.toolpool-gesundheitsforschung.de/). Aus der Zusammenführung aller 15 Kataloge in einer Access-Datenbank wurde ersichtlich, dass es sowohl in der Benennung von Merkmalen als auch bei den Wertemengen identischer Merkmale erhebliche Unterschiede gab. Die Anzahl der Dokumentationseinheiten und Merkmale variierte stark: es wurden zwischen 8 und 126 Dokumentationseinheiten bzw. zwischen 48 und 756 Merkmale in der Konzeptentwicklungsphase definiert ( $\mathbf{A b b}$. 1).

Durch die Überführung der Merkmalskataloge in das Standardmodel der ISO/IEC 11179 wurde u. a. ein Vergleich ermöglicht. In der zweiten Förderphase konnte so jedem der sechs Register ein auf den jeweiligen Merkmalskatalog zugeschnittener Bericht zur Verfügung gestellt werden. Somit wurden die Projekte in die Lage versetzt, ihre Merkmale, Wertemengen und ggf. Kodierungen mit anderen Projekten zu vergleichen und eventuell Änderungen bzw. Ergänzungen vorzunehmen. Die Empfehlungen des Begleitprojekts zu Wertemengen für ausgewählte Merkmale wurden in einigen Projekten umgesetzt, z. B. beim Merkmal Geschlecht. Ein besonderes Interesse der Projekte bestand auch an Wertemengen zu sozioökonomischen Merkmalen wie Schulabschluss oder berufliche Ausbildung.

\section{Datenqualität}

Die Auswahl von Indikatoren zur Datenqualität erfolgte zunächst hinsichtlich der Kriterien Relevanz und Umsetzbarkeit nach einem standardisierten Schema. Hiermit qualifizierten sich 17 der zu Grunde liegenden 51 Qualitätsindikatoren. Bei zusätzlicher Berücksichtigung des Kriteriums Wichtigkeit erwiesen sich sechs Indikatoren als geeignet. Die Anpassung durch die sechs Projekte der Realisierungsphase ergab ein endgültiges Core-Set von fünf Qualitätsindikatoren, das von allen Registern implementiert wurde ( $\triangleright$ Tab. 2). Im Core-Set waren alle drei Ebenen der Qualität nach Donabedian [17] vertreten: Struktur (= Organisation), Prozess ( = Integrität) und Ergebnis (= Richtigkeit). Damit waren die Aspekte Vollzähligkeit, Vollständigkeit und Richtigkeit im Benchmarking abgedeckt.

Für den ersten Qualitätsbericht konnten fünf der sechs Register eingeschlossen werden, da bei diesen bereits 2020 rekrutiert wurde. Neben einem jährlichen Berichtsmodus wurde für drei Qualitätsindikatoren eine quartalsweise Übermittlung der Kennzahlen festgelegt (TMF-1014, TMF-1018 und TMF 1030).

\section{Diskussion}

- Tab. 3 zeigt den Umfang der Beteiligung an den Angeboten des Begleitprojekts. In der Konzeptentwicklungsphase arbeitete das Begleitprojekt entsprechend einem Top-Down-Ansatz, da bei der Vielzahl und Verschiedenheit der Projektideen eine individuelle Unterstützung nicht sinnvoll erschien. Die mit den Projekten abgestimmten Vorlagen für das Registerprotokoll und den Merkmalskatalog wurden von vielen Projekten genutzt. Eine Verpflichtung hierzu bestand nicht. Auch das Angebot einer externen Beratung durch Experten wurde größtenteils wahrgenommen. Die Gewinnung von Experten im Vorfeld war unkompliziert, sodass jedem

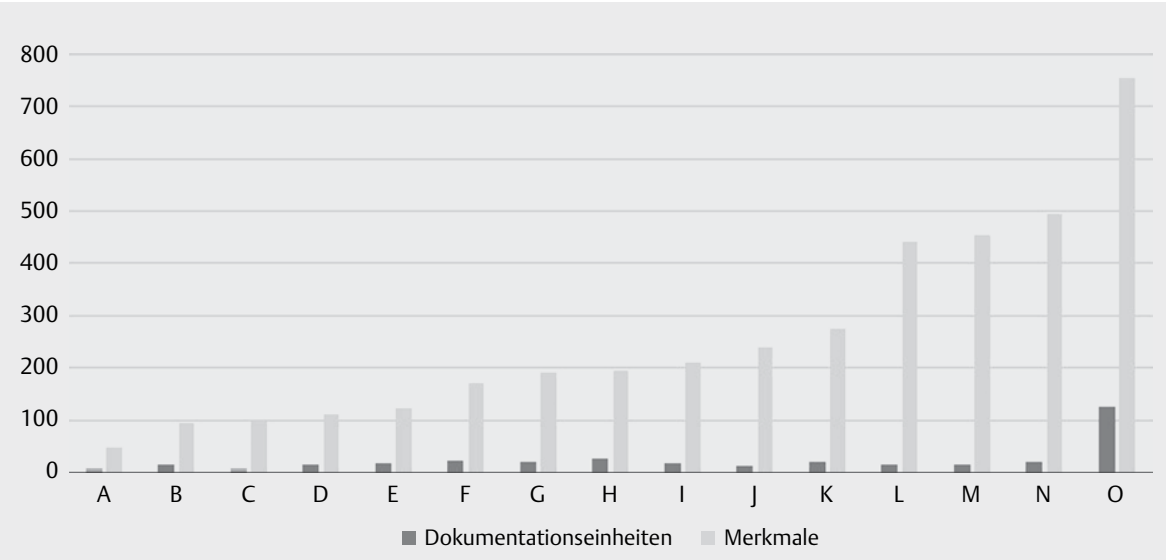

Abb. 1 Anzahl der Dokumentationseinheiten und Merkmale bei 15 Registern. 
Tab. 2 Core-Set der Qualitätsindikatoren.

\begin{tabular}{|l|l|l|}
\hline ID (nach [14]) & Bezeichnung & Ebene \\
\hline TMF-1014 & $\begin{array}{l}\text { Fehlende Werte bei mandatori- } \\
\text { schen Datenelementen }\end{array}$ & Integrität \\
\hline TMF-1018 & $\begin{array}{l}\text { Ausreißer bei stetigen } \\
\text { Datenelementen }\end{array}$ & Integrität \\
\hline TMF-1030 & Rekrutierungsrate & Organisation \\
\hline TMF-1034 & $\begin{array}{l}\text { Vorzeitig ausscheidende } \\
\text { Beobachtungseinheiten }\end{array}$ & Organisation \\
\hline TMF-1046 & Vollständigkeit der Daten & Richtigkeit \\
\hline
\end{tabular}

> Tab. 3 Beteiligung der Projekte.

\begin{tabular}{|l|c|c|c|c|}
\hline Projekt & $\begin{array}{c}\text { Beratung } \\
\text { durch } \\
\text { Experten }\end{array}$ & $\begin{array}{c}\text { Teilnahme } \\
\text { am } \\
\text { IT-Work- } \\
\text { shop }\end{array}$ & $\begin{array}{c}\text { Bereitstel- } \\
\text { lung } \\
\text { Merkmals- } \\
\text { katalog }\end{array}$ & $\begin{array}{c}\text { Auswahl } \\
\text { Quali- } \\
\text { tätsindika- } \\
\text { toren }\end{array}$ \\
\hline A & $\mathrm{x}$ & - & $\mathrm{x}$ & $\mathrm{x}$ \\
\hline B & $\mathrm{x}$ & $\mathrm{x}$ & $\mathrm{x}$ & - \\
\hline C & $\mathrm{x}$ & $\mathrm{x}$ & $\mathrm{x}$ & $\mathrm{x}$ \\
\hline D & $\mathrm{x}$ & - & $\mathrm{x}$ & $\mathrm{x}$ \\
\hline E & - & $\mathrm{x}$ & $\mathrm{x}$ & $\mathrm{x}$ \\
\hline F & $\mathrm{x}$ & $\mathrm{x}$ & $\mathrm{x}$ & $\mathrm{x}$ \\
\hline $\mathrm{G}$ & $\mathrm{x}$ & $\mathrm{x}$ & $\mathrm{x}$ & $\mathrm{x}$ \\
\hline H & $\mathrm{x}$ & - & $\mathrm{x}$ & $\mathrm{x}$ \\
\hline I & $\mathrm{x}$ & $\mathrm{x}$ & $\mathrm{x}$ & $\mathrm{x}$ \\
\hline $\mathrm{J}$ & $\mathrm{x}$ & $\mathrm{x}$ & $\mathrm{x}$ & $\mathrm{x}$ \\
\hline K & $\mathrm{x}$ & $\mathrm{x}$ & $\mathrm{x}$ & $\mathrm{x}$ \\
\hline $\mathrm{L}$ & $\mathrm{x}$ & $\mathrm{x}$ & $\mathrm{x}$ & $\mathrm{x}$ \\
\hline M & $\mathrm{x}$ & $\mathrm{x}$ & $\mathrm{x}$ & $\mathrm{x}$ \\
\hline N & $\mathrm{x}$ & $\mathrm{x}$ & $\mathrm{x}$ & $\mathrm{x}$ \\
\hline O & $\mathrm{x}$ & - & $\mathrm{x}$ & $\mathrm{x}$ \\
\hline P & $\mathrm{x}$ & $\mathrm{x}$ & - & $\mathrm{x}$ \\
\hline
\end{tabular}

Projekt zwei Experten vorgeschlagen werden konnten. Hinzuweisen bleibt auf das Spannungsfeld zwischen der Erfüllung von Ausschreibungskriterien des Projektträgers und einer fachlichen Einschätzung.

Bezüglich der Auswahl einer Softwarelösung war das Ziel des Workshops, die Projekte in die Lage zu versetzen, eine informierte Entscheidung zu treffen. Einige der Projekte hatten zu diesem Zeitpunkt bereits eine Vorentscheidung bezüglich der zu verwendenden Software getroffen. Zum Teil gab es bereits Vorprojekte mit entsprechenden Festlegungen, zum Teil existierten Vorgaben durch Projektpartner der Register. Dass sich schließlich keines der Projekte in der Realisierungsphase für eine der auf dem Workshop vorgestellten Software entschied, deutet weitere Faktoren an, die Einfluss auf die Entscheidung für eine IT-Lösung hatten.

Für die Planung eines Registers spielt die Auswahl der zu erfassenden Merkmale eine große Rolle. Die Auswertung dieser Merkmale führt schließlich zur Beantwortung der wissenschaftlichen Fragestellungen. Um den immer wichtiger werdenden FAIR-Prinzipien gerecht zu werden, ist es sinnvoll, die Metadaten des Registers standardisiert und transparent zu definieren [9]. Dies schließt eine Veröffentlichung der Metadaten ein. Durch die Vorlage für Merkmalskataloge wurde dieses Vorgehen unterstützt. Die vom
Begleitprojekt angestrebte Harmonisierung und Standardisierung zwischen den zunächst 15 und später 6 Registern konnte allerdings nur für wenige Merkmale erreicht werden. Die Sichtung der Merkmalskataloge offenbarte eine große Verschiedenartigkeit der Register mit enormer Variabilität bzgl. Merkmalen und Wertemengen. Auch bei der Verwendung von Standards, Klassifikationen und Fragebogeninstrumenten unterschieden sich die Projekte stark. Als ein Teilschritt zu einer öffentlichen Bereitstellung von Informationen gemäß den FAIR-Prinzipien wurden strukturierte Übersichten zu den Registern auf den Webseiten der Arbeitsgruppe Register des DNVF veröffentlicht (s. https://www.dnvf.de/gruppen/agregister.html). Die Darstellung folgt den vom DNVF für ein Register der Register formulierten Empfehlungen [18].

Ein wichtiger Aspekt bei der Beurteilung der Güte eines Registers bzw. der Ergebnisse der wissenschaftlichen Auswertung ist die zugrundeliegende Qualität der Daten [16]. Der Gedanke eines Benchmarkings der Datenqualität zwischen den Registern der Realisierungsphase über gemeinsam festgelegte Qualitätsindikatoren war eine erhebliche Ausweitung der gängigen Methodik [1]. Im Zuge der Vorarbeiten für dieses Benchmarking war jedes Projekt gefordert, sich mit seiner Datenqualität und deren Messbarkeit auseinanderzusetzen. Die dazu etablierten Prozesse können auch innerhalb eines Registers für den Vergleich der Datenqualität von Studienzentren eingesetzt werden. Ein wichtiger Aspekt ist dabei die Festlegung von entsprechenden Maßnahmen bei Qualitätsdefiziten [19].

Die Zusammenarbeit mit den Projekten - sowohl in der Konzeptentwicklungs- als auch in der Realisierungsphase - war aus Sicht des Begleitprojekts sehr konstruktiv. Allerdings hat auch hier die Corona-Pandemie Einschnitte nach sich gezogen. Insbesondere wurde der persönliche Austausch zwischen Begleitprojekt und Register blockiert. Die Inaugenscheinnahme eines Registers vor Ort kann durch Videokonferenzen kaum ersetzt werden. Prinzipiell stand das Begleitprojekt vor der Herausforderung, zwischen einer Etablierung und Anwendung allgemeingültiger Vorgehensweisen und einer pragmatschen Unterstützung abzuwägen. Derzeit fehlen vielfach noch konkrete Handlungshilfen für die gut abgesicherten, methodischen Empfehlungen [1, 16]. Es konnten daher bislang nur für ausgewählte Aspekte Materialien für eine breite, nachgelagerte Nutzung in der Versorgungsforschung vorgelegt werden.

Das Begleitprojekt kann in seiner Konzeption und Umsetzung selbst als modellhaft verstanden werden. Die Unterstützung von Forschungsprojekten durch eine Begleitstruktur steht im Spannungsfeld von eigenen wissenschaftlichen Interessen und einer administrativen Funktion. Ersteres mag vor allem bei Umsetzung der Begleitstruktur durch eine eigenständige wissenschaftliche Einrichtung befürchtet werden, bei der die Projekte einer Förderinitiative nicht als Auftrag, sondern als Material verstanden werden. Letzteres ergibt sich leicht bei Umsetzung der Begleitstruktur jenseits der wissenschaftlichen Forschung, bei der weniger Unterstützung und wissenschaftliche Bearbeitung als vielmehr Selbsterhalt im Vordergrund stehen. Der weitere Verlauf der Förderinitiative sollte daher genutzt werden, um die Übertragbarkeit der Konzeption des Begleitprojekts dieser Förderinitiative als Blaupause für die Versorgungsforschung in den Blick zu nehmen. 


\section{FAZIT FÜR DIE PRAXIS}

Für jedes Projekt mit dem Ziel des Aufbaus eines Registers bietet sich die Verwendung der erarbeiteten Vorlage für ein Registerprotokoll an. Dieses unterstützt die intensive Beschäftigung mit den Zielen und Aufgaben eines Registers und den dafür zu treffenden Entscheidungen. Die ebenfalls entwickelte Vorlage für einen Merkmalskatalog hilft bei der Strukturierung der zu erfassenden Merkmale und Wertemengen im Sinne der FAIR-Prinzipien. Der Einsatz von Indikatoren ermöglicht eine Beurteilung der Datenqualität und schafft die Möglichkeit zum frühzeitigen Eingreifen bei festgestellten Schwachstellen. Auch außerhalb des Rahmens einer öffentlichen Förderinitiative sind eine Zusammenarbeit von Registern und ein Austausch zwischen Registern sinnvoll, unabhängig von Themengebieten und Zielsetzungen.

\section{Danksagung}

Das Begleitprojekt REGISVF-AP-Teilprojekt B wird vom BMBF gefördert unter den Förderkennzeichen 01GY1720B bzw. 01GY1917B. Wir danken den Projekten der Konzeptentwicklungs- und Realisierungsphase für die Zusammenarbeit.

\section{Interessenkonflikt}

Die Autorinnen/Autoren geben an, dass kein Interessenkonflikt besteht.

\section{Literatur}

[1] Gliklich RE, Leavy MB, Dreyer NA. (sr eds). Registries for Evaluating Patient Outcomes: A User's Guide. $4^{\text {th }}$ ed. AHRQ Publication No. 19(20)-EHC020. Rockville, MD: Agency for Healthcare Research and Quality; 2020

[2] Müller D, Augustin M, Banik N et al. Memorandum Register für die Versorgungsforschung. Das Gesundheitswesen 2010; 72: 824-839

[3] Stausberg J, Altmann U. Register und Kohorten. In: Drepper ], Semler SC, Hrsg. IT-Infrastrukturen in der patientenorientierten Forschung. Aktueller Stand und Handlungsbedarf-2016. Verfasst und vorgelegt vom IT-Reviewing-Board der TMF. Berlin: AKA, 2016: 47-78

[4] Stausberg J, Harkener S, Altmann U et al. Process Coverage and Use Case Support of Health Registry Software in Germany. Stud Health Technol Inform 2020; 272: 79-82
[5] Stausberg J, Altmann U, Antony G et al. Registers for Networked Medical Research in Germany: Situation and prospects. Appl Clin Inform 2010; 1: 408-418

[6] Stausberg J, Harkener S, Siddiqui R et al. IT Infrastructure for Registries in Health Services Research: A Market Study in Germany. Stud Health Technol Inform 2018; 251: 183-186

[7] Michalik C, Dress ], Ngouongo S et al. Requirements and tasks of cohorts and registers, the German KoReglT project. Stud Health Technol Inform 2014; 205: 1085-1089

[8] Zaletel M, Kralj M. eds. Methodological guidelines and recommendations for efficient and rational governance of patient registries. Ljubljana: National Institute of Public Health; Slovenia: 2015

[9] Wilkinson MD, Dumontier M, Aalbersberg IJ et al. The FAIR Guiding Principles for scientific data management and stewardship. Sci Data 2016; 3: 160018. Erratum in: Sci Data 2019; 6(1): 6

[10] Leiner F, Haux R. Systematic planning of clinical documentation. Methods Inf Med 1996; 35: 25-34

[11] ISO/IEEC 11179-3. Information Technology - Metadata Registries (MDR), Part 3: Registry Metamodel and Basic Attributes. Edition 3; 2013

[12] Stausberg J, Harkener S. Bridging Documentation and Metadata Standards: Experiences from a Funding Initiative for Registries. Stud Health Technol Inform 2019; 264: 1046-1050

[13] Arts DG, de Keizer NF, Scheffer G]. Defining and improving data quality in medical registries: a literature review, case study, and generic framework. J Am Med Inform Assoc 2002; 9: 600-611

[14] Nonnemacher M, Nasseh D, Stausberg J. Datenqualität in der medizinischen Forschung. Leitlinie zum adaptiven Management von Datenqualität in Kohortenstudien und Registern. 2., aktualisierte und erw. Aufl. Berlin: Medizinisch Wissenschaftliche Verlagsgesellschaft; 2014

[15] Harkener S, Stausberg J, Hagel C et al. Towards a Core Set of Indicators for Data Quality of Registries. Stud Health Technol Inform 2019; 267: 39-45

[16] Stausberg J, Maier B, Bestehorn K et al. Memorandum Register für die Versorgungsforschung: Update 2019. Das Gesundheitswesen 2020; 82: e39-e66

[17] Donabedian A. Evaluating the quality of medical care. The Milbank Memorial Fund Quarterly 1966; 44: 166-206

[18] Stausberg J, Semler S, Neugebauer EAM. Ein Register für Register und Kohorten: Empfehlungen zu Metadaten und Verfahrensregeln. . Das Gesundheitswesen 2014; 76: 865-873

[19] Schmidt CO, Krabbe CEM, Schössow J et al. Quality standards for epidemiologic cohort studies: An evaluated catalogue of requirements for the conduct and preparation of cohort studies. Bundesgesundheitsblatt Gesundheitsforschung Gesundheitsschutz 2018; 61: 65-77 